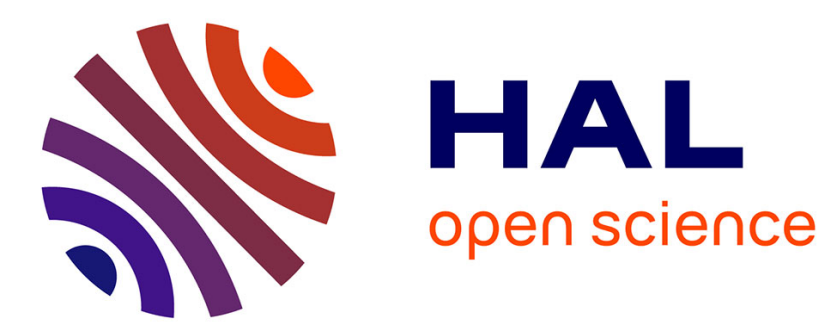

\title{
Revisiting thin film of glassy carbon
}

\author{
Hatem Diaf, António Pereira, Patrice Mélinon, Nicholas Blanchard, Florent \\ Bourquard, Florence Garrelie, Christophe Donnet, Martin Vondráčk
}

\section{To cite this version:}

Hatem Diaf, António Pereira, Patrice Mélinon, Nicholas Blanchard, Florent Bourquard, et al.. Revisiting thin film of glassy carbon. Physical Review Materials, 2020, 4 (6), pp.066002. 10.1103/PhysRevMaterials.4.066002 . hal-02813266

\section{HAL Id: hal-02813266 https://hal.science/hal-02813266}

Submitted on 16 Nov 2020

HAL is a multi-disciplinary open access archive for the deposit and dissemination of scientific research documents, whether they are published or not. The documents may come from teaching and research institutions in France or abroad, or from public or private research centers.
L'archive ouverte pluridisciplinaire HAL, est destinée au dépôt et à la diffusion de documents scientifiques de niveau recherche, publiés ou non, émanant des établissements d'enseignement et de recherche français ou étrangers, des laboratoires publics ou privés. 


\title{
Revisiting thin film of glassy carbon
}

\author{
Hatem Diaf *, Antonio Pereira *, Patrice Melinon *, Nicholas Blanchard *, Florent \\ Bourquard $\uparrow$, Florence Garrelie $\dagger$, Christophe Donnet † and Martin Vondráčk $\ddagger$ \\ *Institut Lumière Matière et CNRS , \\ Université de Lyon, F 69622 Villeurbanne, FRANCE. \\ † Laboratoire Hubert Curien, Université Jean Monnet, 42000 Saint-Etienne, France. \\ $\ddagger$ Institute of Physics, The Czech Academy of Sciences, \\ Na Slovance 2, 182 21 Prague, Czech Republic.
}

(Dated: April 17, 2020) 


\begin{abstract}
Glassy carbon (GC) is a chemically stable form of fully $s p^{2}$-bonded carbon with locally ordered domains. GC is the intermediate material between graphite and diamond combining various properties such as high temperature resistance, hardness, good electrical conductivity, low density, low gases and liquids permeability and excellent resistance to a wide range of aggressive chemical environments. These characteristics make it a very promising material for many applications, but unfortunately it is not widely used because of the high temperatures required for its synthesis. In this work, synthesis of glassy carbon thin films by means of laser ablation of carbon targets under vacuum or in gaseous helium, followed by a nanosecond laser irradiation of the deposited films, is presented. In particular, it is demonstrated that the amorphous structure of a thin film can be efficiently modified to the one of glassy carbon film by nanosecond UV laser irradiation. This method is valuable to prepare thin films similar to commercial glassy carbon with a completely different route which does not require the application of temperature beyond $1000^{\circ} \mathrm{C}$ which is not compatible with the silicon substrate for example. This opens for glassy carbon the way to microengineering applications (mechanics, electronics...). A particular attention is paid to characterize the vitreous carbon. In the literature, the vitreous nature of a carbon layers is often highlighted on the basis of Raman spectroscopy measurements. However, as the Raman spectrum of glassy carbon is similar to that of pyro-carbon, multiwall carbon nanotubes or functional graphene, this technique is not sufficient to safely characterize a carbonaceous material with a high degree of allotropy. To clear up any doubts, additional characterization methods, such as X-ray spectroscopy, transmission electron microscopy and Rutherford backscattering spectrometry, are discussed here.
\end{abstract}

\title{
I. INTRODUCTION
}

Amorphous carbon (a-C) is widely reported in the literature and used in many applications due to the large adaptability of its properties [1-10]. Its structure contains short range ordered domains mixing three hybridizations $s p, s p^{2}$ and $s p^{3}$ with different proportions of each them depending on deposition experimental parameters which in turn determines their properties. The particular case containing an important fraction of $s p^{3}$ is commonly

\footnotetext{
* hatem.diaf@univ-lyon1.fr
} 
known as diamond-like carbon (DLC) or tetrahedral carbon (ta-C) and it is mainly used for protective coatings in various scientific and industrial applications because of its highly mechanical and tribological properties [11].

Post-treatments of as-deposited carbon films are the subject of considerable interest. For example, Ferrari et al [12] showed that conventional thermal annealing under vacuum of ta-C thin films permits to efficiently modify its structure by converting $s p^{3}$ sites to $s p^{2}$ with a drastic change occurring after $1100^{\circ} \mathrm{C}$. More recently, Miyajima et al [10] studied the modification of electrical properties of a-C irradiated by pulsed UV laser and showed an increase in the $s p^{2}$ content with a reduction in resistivity. Such laser based approaches can be carried out in air without carbon oxidation (see Figure S1 Supporting Information), and it permits rapid heating and cooling with precise temporal control. Laser induced $s p^{3}$ to $s p^{2}$ transformation occurs therefore on the order of seconds compared to conventional annealing treatments which take hours [13]. Moreover, the possibility to easily choose the temperature by adjusting the laser parameters and therefore the thermal diffusion depth in the film during the laser treatment permits the formation of $\mathrm{SiC}$ to be avoided and eases the integration of such materials with any kind of substrate. Many studies showed, on the basis of the Raman results, that laser irradiated amorphous carbon thin films have the structure of graphitic clusters or glassy carbon (GC, also called glass-like carbon or vitreous carbon) $[1,14-16]$. In particular, glassy carbon is attracting the interest of materials scientists and industrial companies for its large and original physical properties compared to other carbonaceous materials, such as high electrical conductivity, excellent mechanical resistance and gas impermeability. GC is also biologically compatible with living tissues [17-19]. Finally, GC has the particularity of being the carbonaceous material with the lowest mass density $\left(1.5 \mathrm{~g} / \mathrm{cm}^{3}\right)$ [19] without open porosity, thus combining the properties of vitreous and ceramic materials with those of graphite. For example, it is widely used as an electrode material in electrochemical applications, as well as for high temperature crucible [20]. Glassy carbon is a $s p^{2}$-hybridized, non-graphitisable and isotropic carbon with high structural order. According to the definition of International Union of Pure and Applied Chemistry (IUPAC), GC cannot be described as amorphous carbon. Harris [21-24] has described the glassy carbon as a fully $s p^{2}$-bonded fullerene-related structure which consists of wrinkled fullerenes formed by curved graphene multilayers surrounding closed pores. The presence of this curvature is related to structural defects associated to non-hexagonal rings 
such as pentagons and heptagons [24]. GC is mainly produced by thermochemical decomposition of polymeric resins at high temperature (between 1000 and $2500{ }^{\circ} \mathrm{C}$ ) [20, 25]. High temperature heating of precursor polymers results in an increase of the structural order within the layers as their size increases, as well as the appearance of numerous closed pores. This leads to a decrease in mass density to about $1.5 \mathrm{~g} / \mathrm{cm}^{3}$ and the formation of GC. In the final stage, at high temperature around $3000{ }^{\circ} \mathrm{C}$, GC cannot change to crystalline graphite. Its final microstructure depends on the chemical compositions and structure of the polymer precursors [26]. Even if this approach makes the formation of GC thin films possible, it suffers from many disadvantages: i) the need to work at very high temperatures, which reduces the number of possible substrates, ii) the adhesion to the substrate that can be affected by degassing during pyrolysis and also by the difference in the thermal expansion coefficient between the film and the substrate, and iii) the use of any precursor polymers to form GC causes the film to shrink during pyrolysis [27, 28].

Herein, we report on a simple approach for the synthesis of glass-like carbon thin films at room temperature, which relies on the combination of two laser processes: pulsed laser deposition (PLD) and nanosecond UV laser irradiation. Thanks to an extensive characterization of the irradiated films, we show that after laser irradiation the deposited carbon film show all the features of glassy carbon for a carefully chosen set of process parameters: i) Raman spectra with the two main bands D and $G$ which are situated at around $1330 \mathrm{~cm}^{-1}$ and $1590 \mathrm{~cm}^{-1}$, respectively, together with a $I_{D} / I_{G}$ ratio close to or higher than 1 , and the weak $\mathrm{T}$ band at around $1050 \mathrm{~cm}^{-1}$, ii) X-ray photoelectron (XPS) and Electron Energy loss spectroscopy (EELS) measurements show a high level of $s p^{2}$ hybridization, iii) a low mass density as determined by Rutherford backscattering (RBS), and iv) high resolution transmission electron microscopy (HTREM) images showing curved graphene multilayers surrounding closed pores. On the basis of this investigation, the formation of GC is theoretically discussed.

\section{EXPERIMENTAL SECTION}

Carbon thin films were deposited by PLD at room temperature by means of a pulsed KrF excimer laser (Lambda Physik, $\lambda=248 \mathrm{~nm}$, pulse duration $\tau=20 \mathrm{~ns}$ and repetition rate $\mathrm{f}=10 \mathrm{~Hz}$ ) and by using a commercially vitreous carbon (Neyco, $99.9 \%$ purity, 1.54 
$\mathrm{g} / \mathrm{cm}^{3}$ mass density, noted c.t.). Prior to deposition, the PLD chamber was pumped down to a residual pressure of $\sim 5 \cdot 10^{-7}$ mbar. The fluence was fixed to $12 \mathrm{~J} / \mathrm{cm}^{2}$ and the targetsubstrate distance was set to $4 \mathrm{~cm}$. The carbon films were deposited on Si (100) substrate under vacuum $\left(5.10^{-7} \mathrm{mbar}\right)$ or $1 \mathrm{mbar}$ of He. The substrates were cleaned using ultrasound sonification and sequentially in acetone and ethanol and dried using dry nitrogen.

Pulsed laser irradiation of the deposited films was then performed in air using the same $\mathrm{KrF}$ excimer laser. Laser fluences were fixed to $0.15 \mathrm{~J} / \mathrm{cm}^{2}$ for carbon thin films deposited under $\mathrm{He}$ and $1.5 \mathrm{~J} / \mathrm{cm}^{2}$ for the one deposited under vacuum. The advantage of the use of UV laser is that the optical absorption depth is reduced and thereby avoids heating of the substrate. The thermal diffusion length is linked with the duration of the processing laser pulses [29].

Raman spectra of carbon thin film were measured using an ARAMIS spectrometer (Horiba Jobin Yvon). A confocal mode was used to focus the laser beam on a sample surface with its displacement XYZ stage and a $100 \times$ objective of 0.95 numerical aperture. The experiments were carried out with the unpolarized excitation energy of $633 \mathrm{~nm}$ from a HeNe laser. Spectral resolution of around $2 \mathrm{~cm}^{-1}$ was given by an air cooled CCD detector equipped with a grating of 600 lines. $\mathrm{mm}^{-1}$. Prior to data acquisition, the calibration of the spectrometer was made by taking account of the line of the crystalline silicon at 520.5 $\mathrm{cm}^{-1}$. A low laser energy density was used to irradiate the samples to avoid a laser induced change of its structure during the measurements. The Raman signals from lattice vibrations of carbonaceous materials are sensitive to the degree of graphitization and to structural disorder which are determined by the ratio between two bands $I_{D}$ and $I_{G}$. The evolution of $I_{D} / I_{G}$ can give information about structural changes. For all non-irradiated carbon thin films, only the $\mathrm{D}$ and $\mathrm{G}$ bands were fitted using Voigt functions, whereas for irradiated films and GC c.t., two components were added to be better fitted with the same functions: T peak ranging from 1120 to $1200 \mathrm{~cm}^{-1}$ and reported in $[25,30]$, and A peak at around $1540 \mathrm{~cm}^{-1}$ originating from amorphous carbon coupound [25, 30]. This analysis was supplemented by the HRTEM analysis performed using a JEOL JEM-2100 equipped with a High Tilt polepiece operated at $200 \mathrm{kV}$. The surface composition was studied by XPS using NanoESCA (Omicron Nanotechnology) equipped with a monochromatized Al K $\alpha$ X-ray source $(h \nu=$ $1486.7 \mathrm{eV}$ ). The pass energy of the analyzer was fixed at $100 \mathrm{eV}$. The samples were analyzed with a spot size of approximatively $100 \mu \mathrm{m}$. Fitting of the $C_{1 s}$ spectra were preformed 
using Voigt functions with Shirley background. When the asymmetry of the peak was high, Doniach-ŠSunjić peak shape convoluted with Gaussian function was used instead. The EELS measurements were carried out using a hemispherical electron energy analyzer (VG CLAM 4) operating with incident electron energies of $350 \mathrm{eV}$ and $2 \mathrm{keV}$. The mass density of carbon thin film was obtained using ${ }^{4} \mathrm{He}^{+}$ions of $2 \mathrm{MeV}$ energy delivered by the $4 \mathrm{MeV}$ Van de Graaff accelerator. A detailed description of the setup was provided elsewhere [31].

\section{RESULTS AND DISCUSSIONS}

\section{A. Characterization of glassy carbon by Raman spectroscopy}

According to the literature, the most popular technique used to characterize GC is Raman spectroscopy. The first-order Raman spectra of GC exhibit four main peaks between 1000 and $1800 \mathrm{~cm}^{-1}$. Except for ultraviolet laser excitation, they are activated only by the $s p^{2}$ sites because of the resonant excitation of $\pi$ states [32]. The $\mathrm{G}$ band situated at around 1590 $\mathrm{cm}^{-1}$ is known as a feature of graphitic carbonaceous materials and is activated by a single resonant process. It corresponds to an optical phonon mode at the $\Gamma$ Brillouin zone center with $E_{2 g}$ symmetry, i.e. the ordered $s p^{2}$ bonded carbon. In other words, all $s p^{2}$ bound carbon atoms within the layer (hexagonal rings and carbon chains) participate in the stretching mode vibration. The D band at around $1330 \mathrm{~cm}^{-1}$ is due to the breathing mode of $s p^{2}$ atoms in the six-ring carbon structure and is activated by structural disorder which doesn't exist in the perfectly ordered graphitic materials. The physical significance of the D mode was widely discussed and was finally assigned to the double-resonant Raman scattering associated to the TO phonon located near the $\Gamma$ Brillouin zone [33]. The presence of structural defect induces the creation of holes in the bonds, promoting the double resonance scattering of single phonons by electron-hole relaxations [23]. Cançado et al. [34] showed the presence of armchairs and zigzag edges as active defects responsible for the double resonance Raman process and therefore for the increase in intensity of the D band. The primary characteristic feature of $\mathrm{GC}$ is the intensity ratio of the $\mathrm{D}$ and $\mathrm{G}$ bands $\left(I_{D} / I_{G}\right)$ which is expected to be greater than one. In other words, the D band corresponds to the presence of sixfold ring clusters in all nano-sized $s p^{2}$ domains surrounded by the defective edges. Ferrari and Robertson [35] demonstrated that in disordered and amorphous carbons, the Tuinstra and 
TABLE I. Deconvoluted Raman data for the different films. GC(Glassy carbon c.t.),NP (Non porous film at $10^{-6}$ mbar),P (Porous film/1mbar He),MD(Mass density), $\lambda_{D, G}$ and $F W H M_{D, G}$ in $\mathrm{cm}^{-1}, \mathrm{MD}$ in $\mathrm{g} / \mathrm{cm}^{3}$.

\begin{tabular}{lllllll}
\hline \hline & $\lambda_{D}$ & $F W H M_{D}$ & $\lambda_{G}$ & $F W H M_{G}$ & $I_{D} / I_{G}$ & $\mathrm{MD}$ \\
\hline $\mathrm{GC}$ & 1332 & 54 & 1600 & 64 & 2.2 & 1.5 \\
$\mathrm{NP}$ & 1357 & 362 & 1533 & 213 & 0.9 & 1.9 \\
$\mathrm{P}$ & 1350 & 365 & 1530 & 203 & 0.9 & 0.16 \\
\hline \hline
\end{tabular}

Koening relation $I_{D} / I_{G} \sim 1 / L_{a}$, which is valid for crystallite sizes higher than $2 \mathrm{~nm}$, does not correspond to the development of the $\mathrm{D}$ band related to the growth of hexagonal rings and the increase in defects. They proposed a new relation $I_{D} / I_{G} \propto L_{a}^{2}$ for defective carbons containing small crystallites below $2 \mathrm{~nm}$. The D' band at about $1605 \mathrm{~cm}^{-1}$ is not easily observable and is due to the lattice vibration of graphene multilayers or small graphitic domains which are found in defective graphitic carbon. Thus, this band is also related to the presence of structural defects in $s p^{2}$ carbon materials such as D band but it is governed by intra-valley diffusion and not inter-valley diffusion as for D band. Finally, the T band, observed under UV excitation for tetrahedral amorphous carbon in the 1000-1300 $\mathrm{cm}^{-1}$ range, is due to the $\mathrm{C}-\mathrm{C} s p^{3}$ vibration [36]. But this position is also observable for GC under visible excitation and we assume that such $\mathrm{T}$ band could also originate from the vibrations of non-hexagonal ring carbon defects (i.e., heptagons, pentagons, octagons)[33, 37].

\section{B. Deposition of thin films by nanosecond pulsed laser ablation}

As shown in Figure 1, the carbon film morphology is influenced by the nature of the atmosphere during deposition. As expected, under vacuum (Figure 1a), the film is smooth, uniform and compact with no appearance of porosity. For deposition under He atmosphere (Figure 1b), a porous film is clearly observed, with a cauliflower-like topology. With the increasing background pressure, a plume confinement and therefore a decrease in the velocity of the ablated species occurs, leading to the formation of a porous films [38, 39]. This modification of carbon film morphology is in agreement with RBS measurements showing that the mass density of the film decreases from $1.9 \mathrm{~g} / \mathrm{cm}^{3}$ to $0.16 \mathrm{~g} / \mathrm{cm}^{3}$ when the back- 

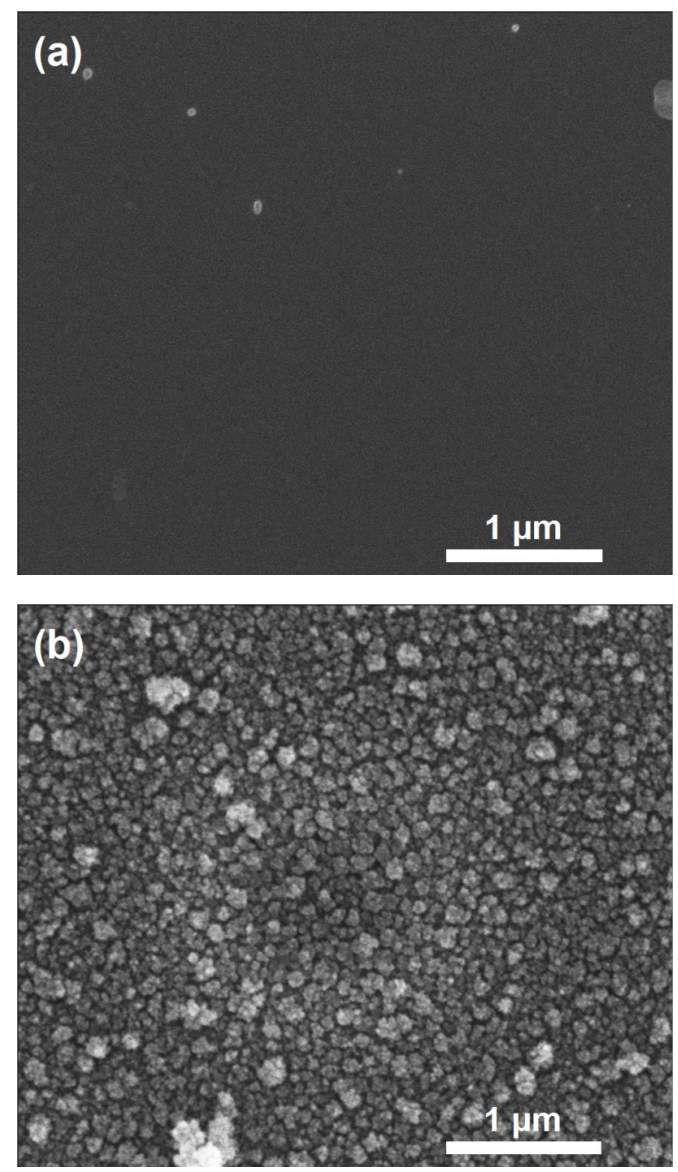

FIG. 1. SEM images of carbon film deposited by PLD at (a) $10^{-6}$ mbar and (b) 1 mbar of helium. 
ground pressure increases from vacuum to 1 mbar of He (Table I). Raman spectra of carbon thin films deposited by PLD as well as the spectrum of GC c.t. for comparison, are shown in Figure 2a-c. All the films deposited by PLD from GC c.t. exhibit amorphous carbon structure. The film deposited under vacuum (Figure $2 \mathrm{~b}$ ) shows spectrum with visible D peak less asymmetric than the one deposited under He (Figure 2c). The D and G intensity ratio is used as a tool to estimate the size of graphitic domains and is also used to obtain information on the carbon structure such as the degree of graphitization and the amount of disorder. To extract such quantitative information, the experimental spectra were fitted using Voigt functions for the $\mathrm{D}$ and $\mathrm{G}$ bands respectively. The results of the Raman spectra fitting (i.e., positions and full width at half maximum of D and G bands and the $I_{D} / I_{G}$ ratio) are summarized in the Table I. The $I_{D} / I_{G}$ ratios are similar for the porous and non-porous carbons films, indicating that they contains the same amount of hexagonal rings. Moreover the $F W H M_{G}$ and $F W H M_{D}$ values for porous and non-porous film are not significantly different. In conclusion, we assume that the effect of helium pressure is weak on the amorphous carbon structure but significant on the mass density [40].

\section{Irradiation of thin film by UV nanosecond pulsed laser}

The amorphous carbon layers were irradiated in air at the optimal fluence threshold $\left(F_{t h}\right)$ of $0.15 \mathrm{~J} / \mathrm{cm}^{2}$ and $1.5 \mathrm{~J} / \mathrm{cm}^{2}$ for the films deposited at $1 \mathrm{mbar}$ of helium (porous film) and under vacuum (non-porous film), respectively (see Experimental section for more details). These two different values of fluence are due to the difference in mass density of the asdeposited layers (see Table 1). Indeed, the denser the matter, the higher irradiation energy is needed to induce structural change. For a better comparison, we therefore determined the laser fluence allowing us to observe structural changes without damaging the layer ( up to around 200 pulses $)$. Since the porous film $\left(0.16 \mathrm{~g} / \mathrm{cm}^{3}\right)$ is almost ten times less dense than the non-porous film $\left(1.9 \mathrm{~g} / \mathrm{cm}^{3}\right)$, it requires about ten times less laser energy. Figure 3 shows the evolution of the Raman spectra of porous carbon films with the number laser pulses. More details on the data processing procedure are included in the Supporting Information (Figure S2). It can be noted that the D and G peaks become more distinct and separate as the number of laser pulses increases. The optimal Raman spectrum, which corresponds to the highest possible $I_{D} / I_{G}$ ratio and the finest possible peaks, is obtained with 200 laser 


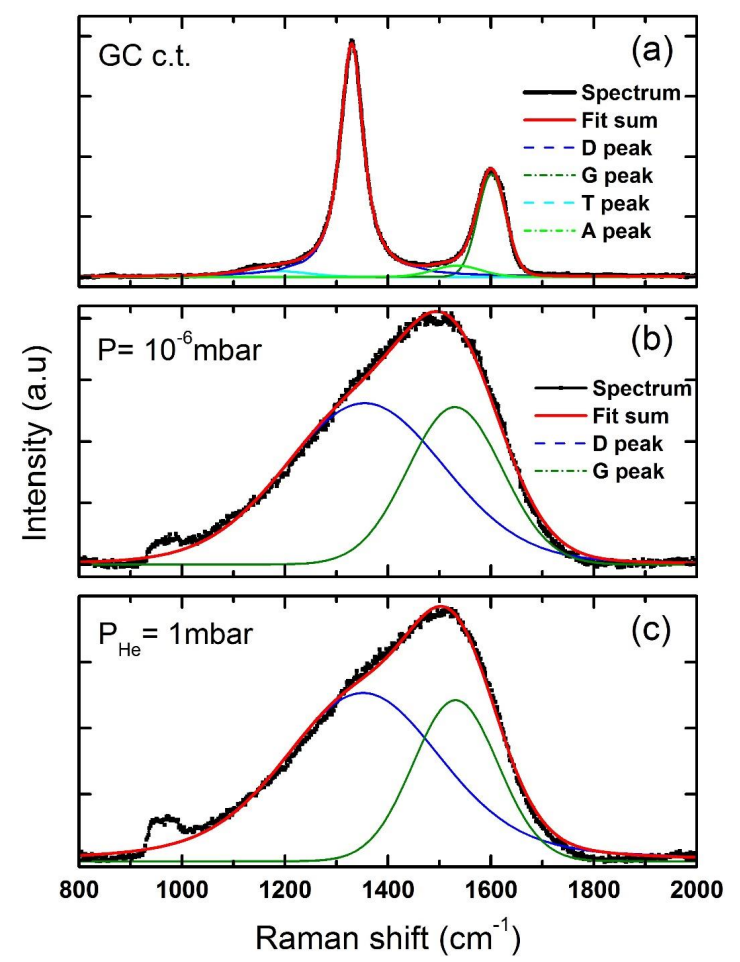

FIG. 2. Raman spectra of the glassy carbon c.t. (a) and of carbon thin films deposited by PLD at two different pressures: (b) $10^{-6}$ mbar and (c) 1 mbar of helium. The peak at $950 \mathrm{~cm}^{-1}$ is due to the second order Raman band from the Si substrate. The red solid line is the result of the fit.

pulses. The Raman peak of silicon did not disappear with the $s p^{3}$ and $s p^{2}$ transition and its intensity remains with an increase in porosity. Above this value, the spectra remain the same except for an increase in peak intensity at $960 \mathrm{~cm}^{-1}$ (silicon substrate), indicating a decrease in the thickness of the carbon layer and therefore its ablation. A similar trend is observed for the non-porous film, for which an optimal Raman spectrum is obtained from 50 laser pulses (see Supporting Information, Figure S2). In this case, it is assumed that the disappearance of the silicon peak with $s p^{3}-\mathrm{sp}^{2}$ transition is due to a change in the optical properties of the non-porous layer. Similar observation can be made comparing graphite, which is completely $s p^{2}$-bound and absorbs visible light, to diamond, which is $s p^{3}$-bound and transparent. Figure 4 shows the evolution of the position and FWHM of D and G bands as well as the $I_{D} / I_{G}$ ratio as a function of the number of laser pulses. As the number of laser pulses increases, the $\mathrm{G}$ band shifted to the higher wavenumbers at $1600 \mathrm{~cm}^{-1}$ whereas 


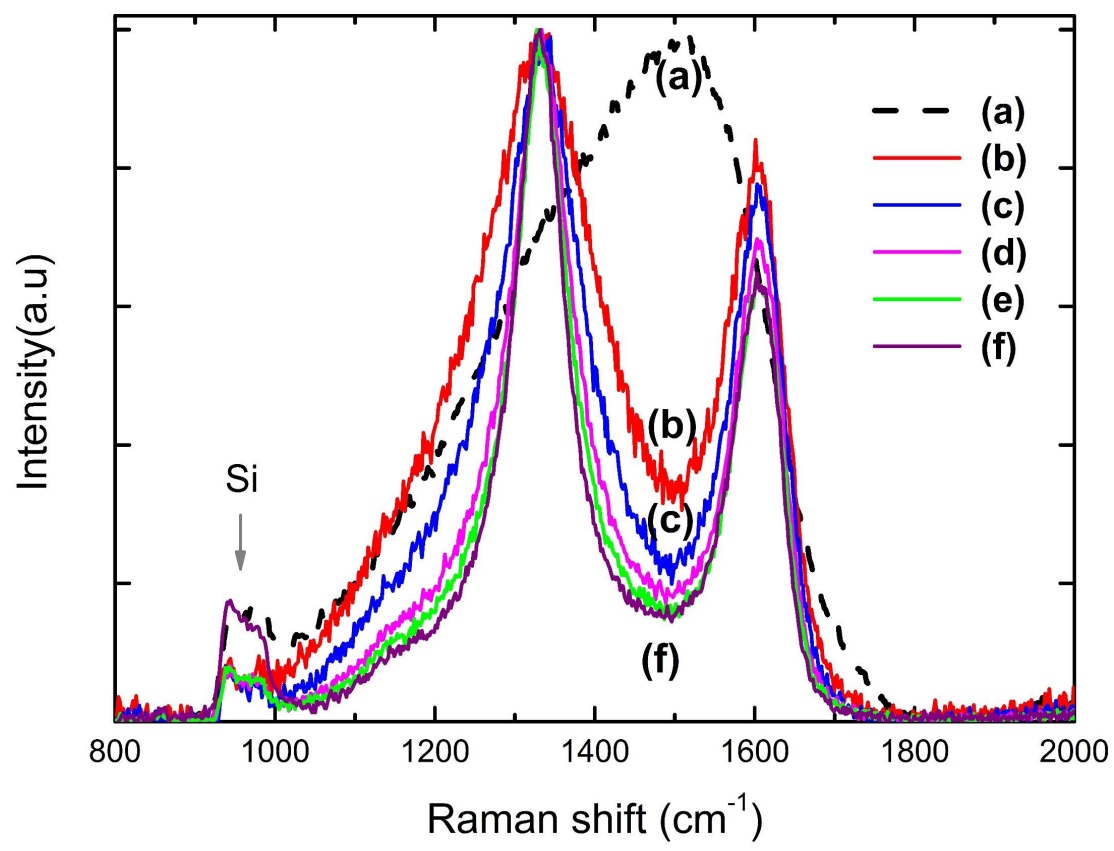

FIG. 3. Corrected Raman spectra of irradiated porous carbon film with different number of laser pulses : (a) un-irradiated carbon, (b) 5 shots, (c) 10 shots, (d) 100 shots, (e) 200 shots, (f) 1000 shots. See Supporting Information [Figure S2] for Raman spectra treatment.

the $\mathrm{D}$ band moved down to $1333 \mathrm{~cm}^{-1}$. The $F W H M_{G}$ and $F W H M_{D}$ narrowed down to $70 \mathrm{~cm}^{-1}$ and $90 \mathrm{~cm}^{-1}$, respectively and correlatively the $I_{D} / I_{G}$ value increased up to 1.85 . The decreasing of $F W H M_{G}$ indicates the increase of graphitic stacking of carbon layer with increase in the crystallites under laser irradiation (the correlation of $F W H M_{D}$ with the level of graphitization is not well understood). The evolution of these parameters exhibits the order in the carbon structure containing defects. Laser irradiation of non-porous carbon film leads also to very similar results (see Supporting Information Figure S2, fitting results not shown here). This indicates that even if both kinds of deposited films are morphologically different at the macroscopic scale, they have the same local structure at the microscopic scale. The values of Raman results indicate the induced-UV laser ordered structural modification and tend to the expected features of glassy carbon.

As visible Raman spectroscopy mainly provides information on the $s p^{2}$ component, it is not possible to evaluate the $s p^{2} / \mathrm{sp}^{3}$ ratio. However, such information can be obtained by XPS after deconvolution of the carbon peak and by calculating the peak area ratio corresponding to $s p^{2}$ and $s p^{3}$ components. High resolution XPS spectra in the $C_{1 s}$ region 

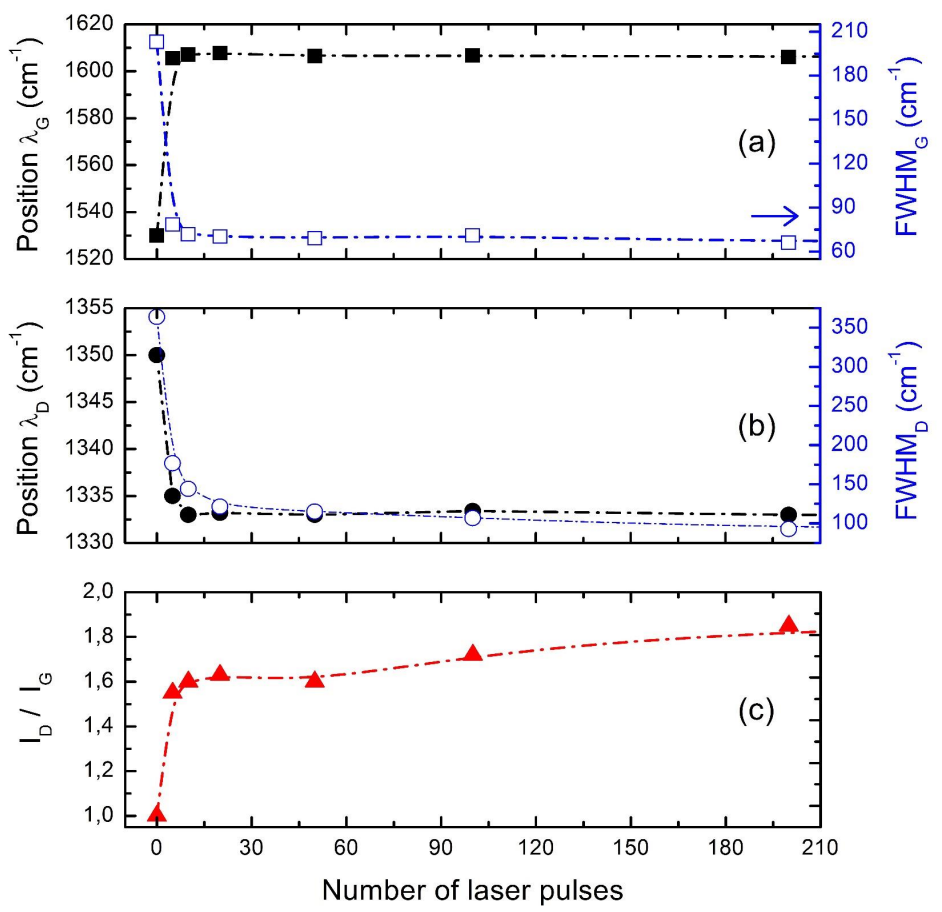

FIG. 4. Characteristics (position and FWHM) of the G (a) and D (b) bands versus the number of laser pulses delivered on porous carbon film deposited by PLD at 1 mbar of He. The $I_{D} / I_{G}$ ratio is given in (c). For the GC c.t., the two bands D and G are situated at around $1330 \mathrm{~cm}^{-1}$ and $1599 \mathrm{~cm}^{-1}$ respectively, and the $I_{D} / I_{G}$ ratio is 2.19 .

of as deposited and irradiated carbon films are shown in Figure 5b,c. For comparison, the XPS spectrum of the glassy carbon c.t. is also reported in Figure 5a. In all three cases, the $C_{1 s}$ spectrum displays two main components located at binding energy of $284.7 \pm 0.2$ and $285.5 \pm 0.2 \mathrm{eV}$ which are assigned to $s p^{2}(\mathrm{C}=\mathrm{C}$ bonds) known as the single feature of the perfect HOPG (Highly Oriented Pyrolytic Graphite) and $s p^{3}$ (C-C) hybridizations states seen as the signature of C-bonds in diamond, respectively. These values are consistent with the literature [41]. To obtain a perfect fit, additional components at $286.1 \mathrm{eV}(\mathrm{C}-\mathrm{O}), 287.2$ $\mathrm{eV}(\mathrm{C}=\mathrm{O})$ and $289.0 \mathrm{eV}(\mathrm{O}-\mathrm{C}=\mathrm{O})$ must be added. Therefore, the $s p^{3}$ hybridization state does not reflect the feature of GC widely viewed as fully $s p^{2}$ bonded carbon. This peak is in fact related to structural defect with distorted $s p^{2}$ orbitals (lattice distortion, vacancies and epoxy groups) [42-46]. For example, Barinov et al. [43] argued on the basis of density functional theory (DFT) calculations that the $C_{1 s}$ component between 285.3 and $285.9 \mathrm{eV}$ should be added because of defective graphite. For the as-deposited porous carbon film at 1 


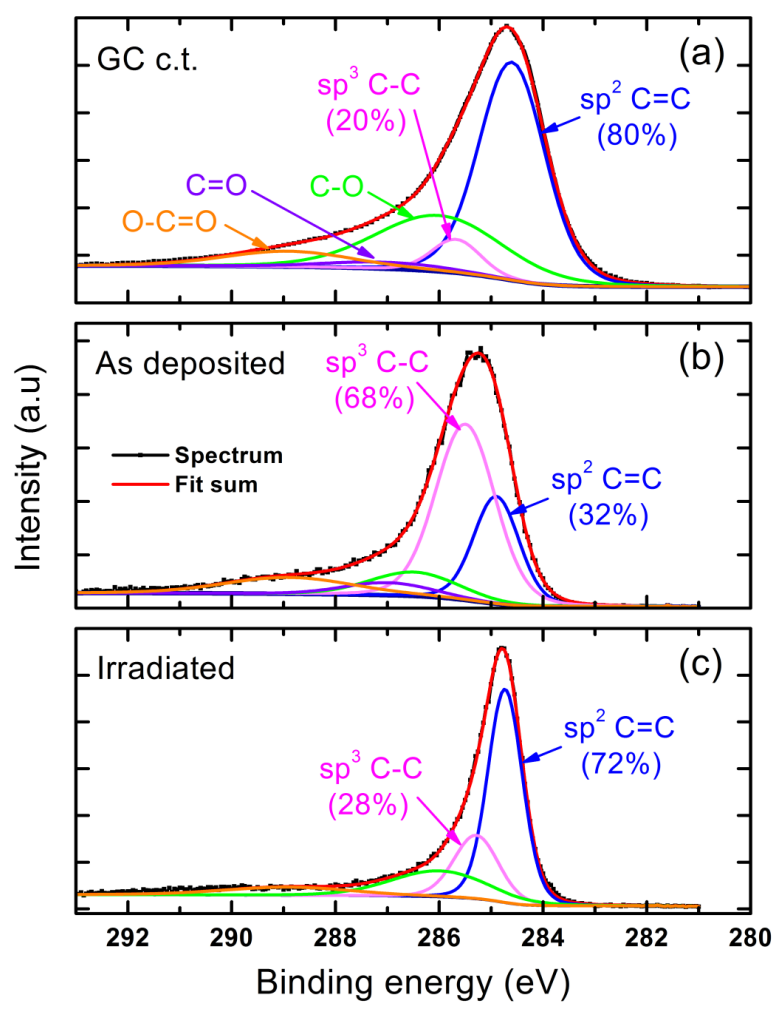

FIG. 5. High resolution XPS spectra of the $C_{1 s}$ peak: (a) glassy carbon c.t., (b) as-deposited carbon film (1 mbar of He), and (c) irradiated film with 1000 laser pulses.

mbar the results of the curve fitting show a mixture of $s p^{2}$ and $s p^{3}$ hybrid states with high fraction of $s p^{3}$ sites (68\%) which is seen as diamond like carbon. The laser irradiation of the carbon film results in a significant change in its hybridization state, with a $s p^{2}$ rate that increases from around $32 \%$ to $72 \%$. It can be noted that even if these results are in line with the Raman analyses, the $s p^{2}$ rate remains below that of the carbon c.t. The reason for this probably lies in the fact that this porous film has a higher surface which enhances the degree of defects, especially the chemisorption of oxygen atoms on the surface and consequently increases the defect peak. It should be noted that the defect peak does not decrease and remains unchanged even if the film started to be ablated, indicating that the deposited film is non-graphitisable (e.g., with 500 laser pulses increase of peak intensity peak at 960 $\mathrm{cm}^{-1}$, see Figure 3). This modification of hybridization state with laser irradiation can be explained by the fact that $s p^{3}$ sites require less energy than $s p^{2}$ sites to be dissociated. As a result, $s p^{3}$ sites preferentially change to $s p^{2}$ leading to an ordered carbon structure. These 
results are supported by the EELS measurements (Figure 6) which indicates the presence of a $\pi$-plasmon peak at around $6 \mathrm{eV}$ in the irradiated film. Glassy carbon, known as all $s p^{2}$ bonded carbon also exhibits this same peak located at loss energy of $6 \mathrm{eV}$ corresponding to the collective excitation of $\pi$ electrons $\left(\pi-\pi^{*}\right.$ transitions) and indicating the $s p^{2}$ bonding structure $[42,43]$. The pulsed laser irradiation of the carbon film is accompanied by the bonding modification from amorphous carbon to ordered carbon before the beginning of ablation. We found the same binding change mechanism for both types of carbon films, namely porous and non-porous films. Nevertheless, while the ordered laser-induced structure seems identical in both cases, Figure 1 shows different macroscopic morphologies in these two films before laser irradiation. The SEM images in Figure 7 show the physical effect of laser irradiation on porous film (not visible on non-porous film due to its higher mass density, see Supporting Information Figure S3). It indicates that the laser irradiation induces the formation of holes inside the surface. Such morphological modification is accompanied by a decrease in the density of the layer. This decreases rapidly with the number of pulses to reach a constant value of about $0.09 \mathrm{~g} / \mathrm{cm}^{3}$ after 5 pulses (see Supporting Information, Figure S4). These results are in agreement with the works of Kononenko et al. [47] on laser-induced mechanisms in DLC films showing that the absorbed laser pulse produces an expansion of surface layer and reduces its mass density. Furthermore, Grigonis et al. [5] demonstrated that the conversion of the $s p^{3}$ to $s p^{2}$ sites caused the swelling.

Finally, the results presented so far show that pulsed laser irradiation of a porous and non-porous carbon film is accompanied by an increase in porosity and a modification of the bonds (conversion of the $s p^{3}$ to $s p^{2}$ sites), and leads to a better organized carbon structure. Although all these characteristics tend to demonstrate the formation of GC, they may also correspond to other structures such as pyro-carbon. To validate the formation of GC, other characterization methods, such as low frequency Raman and HRTEM, were performed. Low-frequency Raman analyses were mainly performed on non-porous films, due to the weak signal obtained with porous films (low mass density). However, the latter were used for HRTEM analyses. They are easy to exfoliate, which allows material to be deposited on a TEM grid without any mechanical thinning or ion beam for the preparation. The lowfrequency Raman spectra of non-porous films before and after laser irradiation are shown in Figure 8. The spectrum of the GC c.t. is also given for comparison. In the low-frequency 


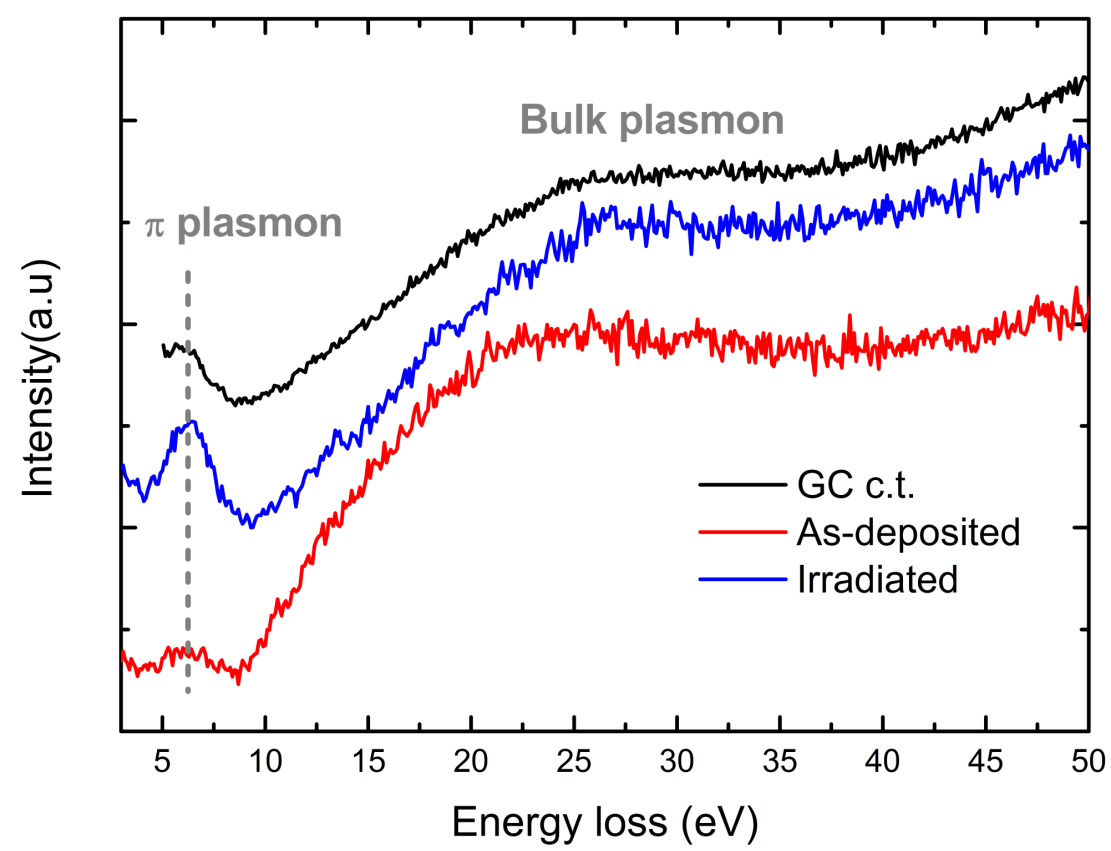

FIG. 6. Electron energy loss spectra of as deposited carbon film (1mbar of He) and irradiated film with 200 laser pulses. Spectrum of the glassy carbon c.t. is also shown for comparison.

region between 150 and $550 \mathrm{~cm}^{-1}$, the two Raman peaks located at 224 and $334 \mathrm{~cm}^{-1}$ are signature of curvature-related structures which are normally present in GC. These peaks are also found for fullerenes, carbon nanotubes, or carbon onion structures, but completely absent in the case of graphite [48]. By now comparing the GC c.t. and the signs of irradiated film (Figure 8a and 8b, respectively), it can be assumed that laser irradiation leads to the formation of heptagons and pentagons in the hexagonal lattice. As reported in the literature, $[37,49-52]$ these non-hexagonal rings induce the curvature in the network and make it isotropic. This structural distortion is observed in the HRTEM image of the irradiated porous-film (i.e., induced $s p^{2}$-like carbon film, Figure 9b), where the stacking graphitic sheets are also visible unlike the amorphous structure before irradiation (Figure 9a). The GC c.t. (Figure 9c) and the $s p^{2}$-like carbon film (Figure 9b) also show curved and facetted carbon sheets with two or three layers that contain voids of 5-10 nm in diameter. More in details, the same two structures (label 2) are wrapped by another graphene at a distance of about $0.32 \mathrm{~nm}$. Its diameter is about $1.0 \mathrm{~nm}$, which is identical to the size of a $C_{60}$ fullerene (label 1). Such fragments, observed in Figure 9b, contain less than 10 graphene sheets and cross in all directions and interconnect with each other. The average inter-layer distance extracted from Figure 9b is about $0.34 \mathrm{~nm}$, which corresponds to turbostratic graphite [46]. 

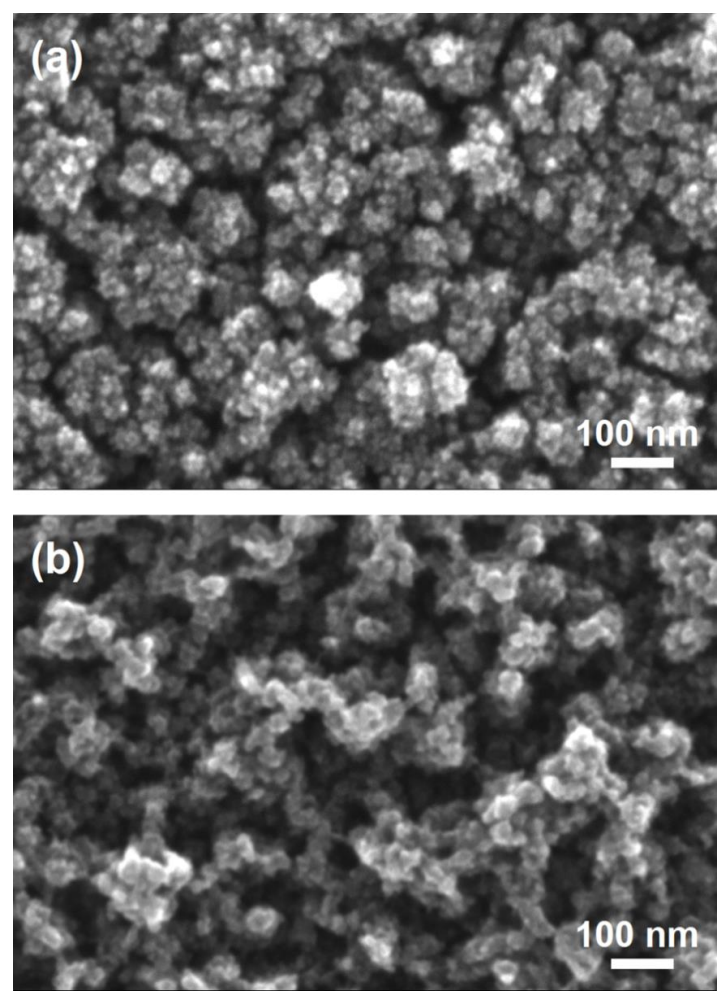

FIG. 7. SEM images of a porous carbon film (deposited at 1 mbar of helium) before (a) and after (b) laser irradiation. 


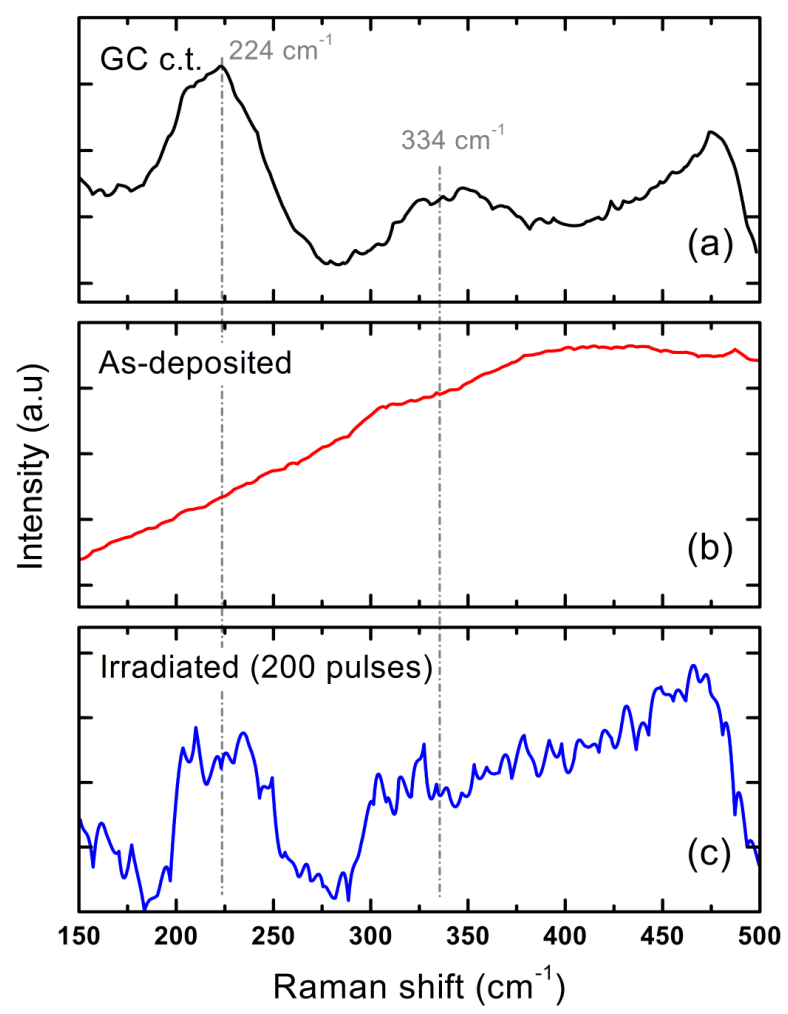

FIG. 8. Low-frequency Raman spectra of the glassy carbon c.t. (a) and the non-porous carbon film before (b) and after laser irradiation with 200 pulse (c).

The intensity profile of these multilayers fragments is shown in the Supporting Information (Figure S5). It is interesting to note that several particles (label 3) have a perfect hexagonal shape, i.e. well-defined facets in the shape of a hexagon with a $120^{\circ}$ curvature angle. Label 4 indicates two regions of negative $(K<0)$ and positive $(K>0)$ curvatures. Finally, the region labeled 5 shows the bifurcation of graphene layers indicating probably a local hyperbolic structure characterized by an inversion of the Gauss curvature (more details in the next section). These results clearly exhibit the strong similarity between glassy carbon and our irradiated carbon films (porous and non-porous). The laser-induced structural modification on the amorphous carbon film results in the formation of the isotropic $s p^{2}$-like structure composed of the mixture of fullerene-like $(\mathrm{K}>0)$, hyperbolic $(\mathrm{K}<0)$ and curved ribbon-like structures which interconnect between them in all directions with the decrease in mass density. All results finally support that the $s p^{2}$-like film is viewed as a glassy-like carbon structure. 

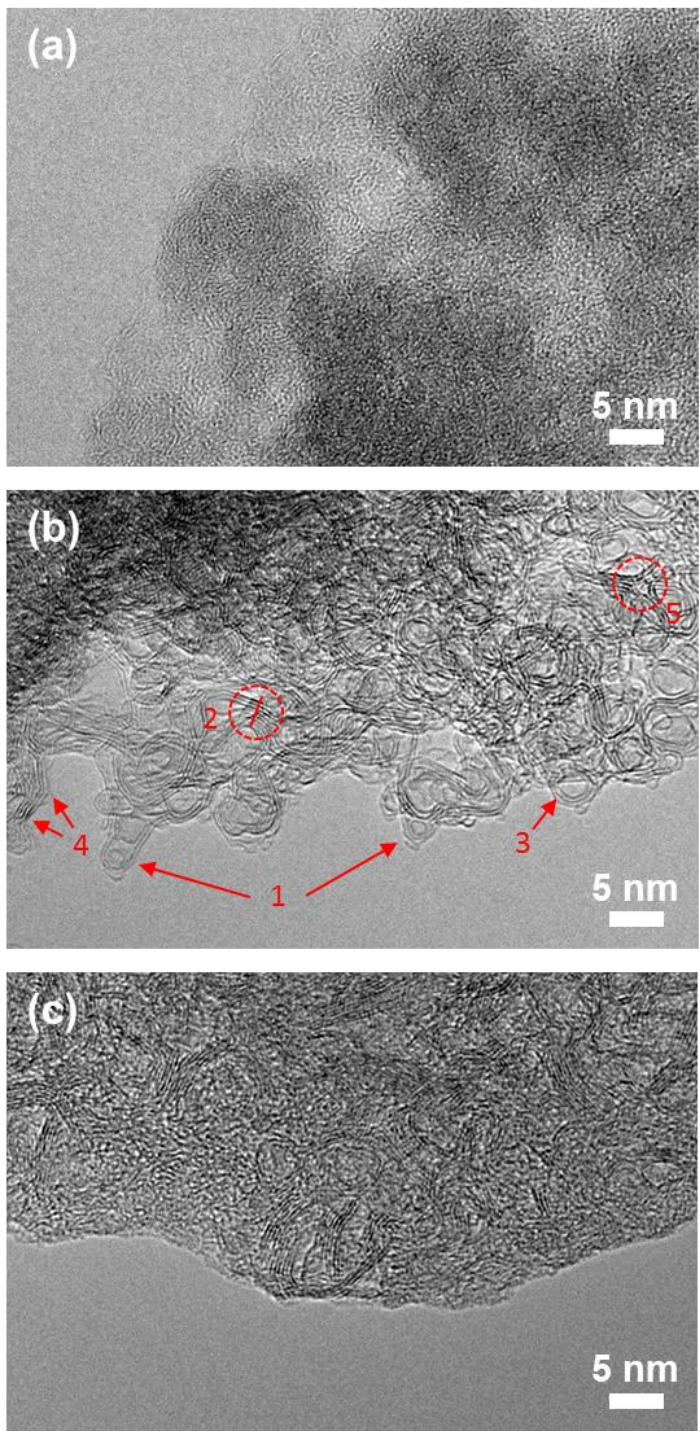

FIG. 9. HRTEM images of porous carbon film: (a) before irradiation and (b) after irradiation with 200 pulses. (c) corresponds to the glassy carbon c.t.. The labels in (b) correspond to (1) fullerene, (2) multilayers fragment, (3) honeycomb-like fragment, (4) presence of curvature with the angle of 120 and (5) hyperbolic structure. 


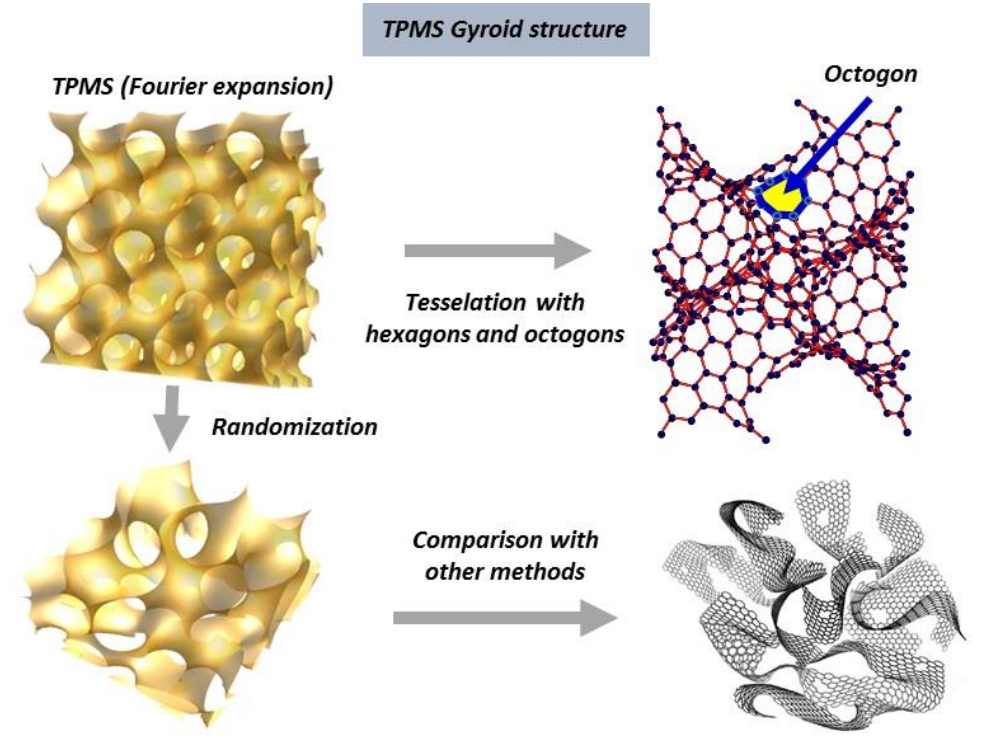

FIG. 10. Different steps to prepare amorphous TPMS structure compared to other methods (right bottom panel) [53]. 


\section{GLASS-LIKE (GL) CARBON STRUCTURE}

The lack of long-range periodicity presents a theoretical challenge. As it is mentioned above, Raman features are not enough to fully characterize glassy carbon. For example, the same $I_{D} / I_{G}$ ratio signature is reported in glassy graphene [54]. But there is one thing we can be sure of: GL is isotropic, porous, stable, metal-like and fully $s p^{2}$ hybridized. Because of the lack of long range order, ab initio calculations performed on large elemental cells are difficult and molecular dynamics based on semi classical potentials are elusive (see for example all the papers dedicated to carbon cluster structure $[55,56])$. However, simple geometrical considerations help us to qualitatively understand the structure. First of all, the $s p^{2}$ hybridized structure suggests a planar sheet which is in mathematics a surface in $E^{2}$ Euclidean space. A porous structure suggests a non-zero genus which is a main characteristic of surfaces in hyperbolic space (negative Gaussian curvature K). The isotropic structure of GL leads to a genus $\mathrm{g} \geq 2$ [57]. Hyperbolic space appears by the introduction of $\mathrm{n}$-gons with $\mathrm{n} \geq 7$ (for example seven-membered or octogonal rings) in the primitive hexagonal structure (graphene) with the creation of saddle points $(K<0)$. Note that spherical space that characterize fullerenes appear by introducing five-membered rings instead of sevenmembered rings (genus in fullerenes is zero). The first prototype which meets all these features named "Schwarzite" was discussed a long time ago [58]. This hypothetic structure is based from triply periodic minimal surfaces (TPMS) (a minimal surface as a zero mean curvature radius) that ensure a cohesive energy not so far from other allotropic forms of carbon. TPMS structures are commonly deduced from differential geometry through the Weierstrass-Enneper relationship or approximated Fourier expansion. Despite the presence of $n$-gons $n \neq 6$ in the structure the strong $s p^{2}$ character is corroborated by the presence of Dirac cones as reported in graphene. A TPMS has no self-intersection and partitions the space into two disjoint labyrinthine regions. Contrary to fullerenes where isolated pentagon rule takes place, it seems that abutting heptagons are more stable [59]. Then, Schwarzites are probably less stable than disordered Schwarzites (abutting heptagons induce disorder in the structure). Experimental evidence of disordered Schwarzite structures was done by Barborini et al. [60], Rode et al. [61] and Wang et al. [62] n-fused rings with $\mathrm{n} \neq 6$ in graphene introduces ripples and then a strain energy. Assuming the lowest strain energy, one has to consider five-membered, seven-membered rings or 8-membered rings no more. 
Applying the Gauss-Bonnet theorem for the genus to a closed orientable surface, and Euler's law it holds

$$
N_{5}-N_{7}-2 N_{8}=12(1-g)
$$

Non isotropic network with positive curvature writes

$$
N_{5}-N_{7}-2 N_{8}=12
$$

Defect free fullerenes lead to $N_{5}=12$. If $N_{7}$ is positive, one observes fullerenes with $(5,7)$ Stone-Wales defects. Isotropic porous structures with $\mathrm{g}=3$ yield:

$$
N_{5}-N_{7}-2 N_{8}=24
$$

The simplest form with $N_{5}=N_{8}=0$ gives the diamond D-type Schwarzite with only heptagons and 28 atoms per elemental cell (for one partition of space $\mathrm{g}=2[63]$ ). Although TPMS is a theoretical object, our feeling is that porous isotropic structure is rich in heptagons (or n-gons with $\mathrm{n}>7$ ). The creation of $(55,77)$ Stone-Wales defects in a pure graphene (formed by rotating a $\mathrm{C}-\mathrm{C}$ bond by $90^{\circ}$ with regard to the midpoint of the bond) comes with a high energy cost (few eV) [50,52, 64, 65], but native pentagonal rings in the graphene structure considerably lowers the barrier for the heptagon formation up to an energy close to few $k_{B} T$ [64]. Since pentagonal rings are present in amorphous carbon with $s p^{2} / s p^{3}$ hybridized states, the GL carbon structure can be formed if the basic structure before annealing is rich in $s p^{3}$ hybridized states. This is corroborated by experiments. Starting with rich graphite-like precursors is not the best way to prepare GL carbon. Conversely, the best that we can do is obtained from $s p^{2} / s p^{3}$ amorphous precursors. Since infinite networks can be built up from basic TPMS structures, the choice of the candidate is arbitrary. However, TPMS structures are either metallic or insulating [66]. Experimental evidence of metal-like character after annealing suggests candidates with metal-like character. Amorphous TPMS structure can be obtained using a standard treatment of the primitive Fourier expansion with a scale distortion that preserves minimality according to Osseman's theorem $[66,67]$. The algorithm is decomposed in three steps (see reference [44]):

- Mathematical structure given by approximate Fourier expansion;

- Tessellation of the surface with hexagons and n-gons $(n=5,7,8)$ that mimics carbon 
structure with a crystallographic group space in $E^{3}$ Euclidean space;

- Randomization with Osserman's method.

Figure 10 displays such an example (gyroid structure with octamers) compared to 3D structure model of the porous 3D graphene using molecular mechanics method [53]. Even though this method relies on purely geometrical considerations, the obtained structure is qualitatively the same as other time-consuming computational methods.

\section{CONCLUSION}

Based on the data from all experimental results using two amorphous carbon film deposited in helium and in vacuum whose microstructures are similar at the nanometer scale but different at the macroscopic level, our in depth investigation shows that amorphous carbon with high $s p^{3}$ rate could be converted to glass-like carbon under ns-laser irradiation with the transition of $s p^{3}$ to $s p^{2}$ sites. $s p^{3}$ sites are seen as the precursor of the glassy-carbonlike formation. In addition, the Raman analysis at low frequency exhibits the formation of the Stone-Wales defects after laser annealing. So, laser irradiation involves two transformation mechanisms: creation of pentagons and heptagons in the hexagonal network and $s p^{3}$-to- $s p^{2}$ phase change. When laser annealing is maintained without ablation for a long time, local graphitization degree increases with a decrease of defects. But as bonding is already established between graphitic fragments in the 3D space, the graphitization cannot be completely reached and some defects remain even at very high temperature, which gives non-graphitisable carbon such as glassy carbon. Moreover, laser irradiation also induces swelling in carbon during irradiation resulting in a decrease in mass density. The TEM observation indicates the coexistence between fullerenes and graphitic nano-crystallites which

interconnect each other by curved sheets of graphene. Such results emphasize the strong resemblance between the microstructure of irradiated carbon film with that of glassy carbon. In conclusion, the irradiated carbon film obtained under theses experimental conditions change to a glassy carbon like one. It is now possible to synthetize a thin film of glassy carbon with only a laser for deposition and irradiation in place of the pyrolysis. This in-depth characterization offers to researchers some possibilities to use the thin film of glass carbon for their research activity and to material engineers for the next-generation applications which benefit from the properties of glassy carbon. 


\section{ACKNOWLEDGMENTS}

We thank Olivier Boisron for EELS measurements. Photoemission experiments were performed at the NanoESCA facilities supported by MEYS of the Czech Republic through project SOLI D21CZ.02.1.01/0.0/0.0/16_019/0000760

[1] N. R. Arutyunyan, M. S. Komlenok, E. V. Zavedeev, and S. M. Pimenov, Physica Status Solidi (b) 255, 1700225 (2018).

[2] W. Hurler, M. Pietralla, and A. Hammerschmidt, Diamond and Related Materials 4, 954 (1995).

[3] A. J. Bullen, K. E. OHara, D. G. Cahill, O. Monteiro, and A. Von Keudell, Journal of Applied Physics 88, 6317 (2000).

[4] W. Duley, The Astrophysical Journal 287, 694 (1984).

[5] A. Grigonis, L. Marcinauskas, V. Vinciunaite, and G. Raciukaitis, Open Physics 9, 1344 (2011).

[6] J. Diaz, G. Paolicelli, S. Ferrer, and F. Comin, Physical Review B 54, 8064 (1996).

[7] Y. Lifshitz, Diamond and Related Materials 12, 130 (2003).

[8] N. D. Baydoğan, Materials Science and Engineering: B 107, 70 (2004).

[9] S. Adhikari, S. Adhikary, A. M. Omer, M. Rusop, H. Uchida, T. Soga, and M. Umeno, Diamond and Related Materials 15, 188 (2006).

[10] Y. Miyajima, A. Adikaari, S. Henley, J. Shannon, and S. Silva, Applied Physics Letters 92, 152104 (2008).

[11] B. Tay, X. Shi, H. Tan, H. Yang, and Z. Sun, Surface and Coatings Technology 105, 155 (1998).

[12] A. Ferrari, B. Kleinsorge, N. Morrison, A. Hart, V. Stolojan, and J. Robertson, Journal of Applied Physics 85, 7191 (1999).

[13] J. Abrahamson, R. Vander Wal, P. Gokulakrishnan, R. Joklik, and C. Fuller, in TechConnect Briefs 2018 - Materials for Energy, Efficiency and Sustainability, Vol. 2 (TechConnect, 2018) pp. 105-108. 
[14] E. Cappelli, C. Scilletta, S. Orlando, V. Valentini, and M. Servidori, Applied Surface Science 255, $5620(2009)$.

[15] L. Marcinauskas, A. Grigonis, P. Valatkevicius, and A. Medvid, Applied Surface Science 261, 488 (2012).

[16] Á. Mechler, P. Heszler, Z. Kántor, T. Szörényi, and Z. Bor, Applied Surface Science 138, 174 (1999).

[17] M. Vomero, E. Zucchini, E. Delfino, C. Gueli, N. Mondragon, S. Carli, L. Fadiga, and T. Stieglitz, Materials 11, 2486 (2018).

[18] R. Mishra, B. Pramanick, T. K. Maiti, and T. K. Bhattacharyya, Microsystems \& Nanoengineering 4, 38 (2018).

[19] M. Hassler, in Coatings for biomedical applications (Elsevier, 2012) pp. 75-105.

[20] P. Harris, Philosophical Magazine 84, 3159 (2004).

[21] P. J. Harris, Journal of Materials Science 48, 565 (2013).

[22] P. J. Harris, International Materials Reviews 42, 206 (1997).

[23] P. J. Harris and S. C. Tsang, Philosophical Magazine A 76, 667 (1997).

[24] P. J. Harris, Z. Liu, and K. Suenaga, Journal of Physics: Condensed Matter 20, 362201 (2008).

[25] K. Jurkiewicz, M. Pawlyta, D. Zygadło, D. Chrobak, S. Duber, R. Wrzalik, A. Ratuszna, and A. Burian, Journal of Materials Science 53, 3509 (2018).

[26] S. Sharma, C. S. Kumar, J. G. Korvink, and C. Kübel, Scientific Reports 8, 16282 (2018).

[27] A. Singh, J. Jayaram, M. Madou, and S. Akbar, Journal of the Electrochemical Society 149, E78 (2002).

[28] B. Y. Park, L. Taherabadi, C. Wang, J. Zoval, and M. J. Madou, Journal of the Electrochemical Society 152, J136 (2005).

[29] J. Fontaine, C. Donnet, and A. Erdemir, in Tribology of diamond-like carbon films (Springer, 2008) pp. 139-154.

[30] A. Sadezky, H. Muckenhuber, H. Grothe, R. Niessner, and U. Pöschl, Carbon 43, 1731 (2005).

[31] D. Pelloux-Gervais, Caractérisation par méthodes nucléaires avancées de boîtes quantiques d'In (Ga) As épitaxiées sur silicium, Ph.D. thesis (2012).

[32] A. C. Ferrari and J. Robertson, Philosophical Transactions of the Royal Society of London. Series A: Mathematical, Physical and Engineering Sciences 362, 2477 (2004).

[33] M. Couzi, J.-L. Bruneel, D. Talaga, and L. Bokobza, Carbon 107, 388 (2016). 
[34] L. Cancado, M. Pimenta, B. Neves, M. Dantas, and A. Jorio, Physical Review Letters 93, 247401 (2004).

[35] A. C. Ferrari and J. Robertson, Physical Review B 61, 14095 (2000).

[36] K. Gilkes, H. Sands, D. Batchelder, J. Robertson, and W. Milne, Applied Physics Letters 70, 1980 (1997).

[37] T. Fujimori, L. R. Radovic, A. B. Silva-Tapia, M. Endo, and K. Kaneko, Carbon 50, 3274 (2012).

[38] E. Irissou, B. Le Drogoff, M. Chaker, M. Trudeau, and D. Guay, Journal of Materials Research 19, 950 (2004).

[39] A. Pereira, T. Martin, M. Levinta, and C. Dujardin, Journal of Materials Chemistry C 3, 4954 (2015).

[40] M. Rusop, S. Mominuzzaman, T. Soga, T. Jimbo, and M. Umeno, International Journal of Modern Physics B 16, 866 (2002).

[41] P. K. Chu and L. Li, Materials Chemistry and Physics 96, 253 (2006).

[42] H. Estrade-Szwarckopf, Carbon 42, 1713 (2004).

[43] A. Barinov, O. B. Malcioglu, S. Fabris, T. Sun, L. Gregoratti, M. Dalmiglio, and M. Kiskinova, The Journal of Physical Chemistry C 113, 9009 (2009).

[44] D. Lee and J. Seo, The Journal of Physical Chemistry C 115, 2705 (2011).

[45] R. Larciprete, S. Gardonio, L. Petaccia, and S. Lizzit, Carbon 47, 2579 (2009).

[46] R. Larciprete, S. Fabris, T. Sun, P. Lacovig, A. Baraldi, and S. Lizzit, Journal of the American Chemical Society 133, 17315 (2011).

[47] T. Kononenko, S. Pimenov, V. Kononenko, E. Zavedeev, V. Konov, G. Dumitru, and V. Romano, Applied Physics A 79, 543 (2004).

[48] S. Bukalov, L. Leites, A. Sorokin, A. Kotosonov, et al., Nanosystems: Physics, Chemistry, Mathematics 5, 186 (2014).

[49] T. Ichihashi, Y. Ando, et al., Nature 356, 776 (1992).

[50] J. Ma, D. Alfè, A. Michaelides, and E. Wang, Physical Review B 80, 033407 (2009).

[51] T. Dinadayalane, J. S. Murray, M. C. Concha, P. Politzer, and J. Leszczynski, Journal of Chemical Theory and Computation 6, 1351 (2010).

[52] J. Kotakoski, J. Meyer, S. Kurasch, D. Santos-Cottin, U. Kaiser, and A. Krasheninnikov, Physical Review B 83, 245420 (2011). 
[53] L. Zhang, F. Zhang, X. Yang, G. Long, Y. Wu, T. Zhang, K. Leng, Y. Huang, Y. Ma, A. Yu, et al., Scientific reports 3, 1408 (2013).

[54] X. Dai, J. Wu, Z. Qian, H. Wang, J. Jian, Y. Cao, M. H. Rummeli, Q. Yi, H. Liu, and G. Zou, Science Advances 2, e1601574 (2016).

[55] D. McCulloch, D. McKenzie, and C. Goringe, Physical Review B 61, 2349 (2000).

[56] J. Jiang and S. I. Sandler, Journal of the American Chemical Society 127, 11989 (2005).

[57] W. H. Meeks III, Bulletin of the American Mathematical Society 83, 134 (1977).

[58] S. Gaito, L. Colombo, and G. Benedek, EPL (Europhysics Letters) 44, 525 (1998).

[59] K. Yoon, A. Rahnamoun, J. L. Swett, V. Iberi, D. A. Cullen, I. V. Vlassiouk, A. Belianinov, S. Jesse, X. Sang, O. S. Ovchinnikova, et al., ACS Nano 10, 8376 (2016).

[60] E. Barborini, P. Piseri, P. Milani, G. Benedek, C. Ducati, and J. Robertson, Applied Physics Letters 81, 3359 (2002).

[61] A. V. Rode, S. Hyde, E. Gamaly, R. Elliman, D. McKenzie, and S. Bulcock, Applied Physics A 69, S755 (1999).

[62] Z. Wang, L. Yu, W. Zhang, J. Han, Z. Zhu, G. He, Y. Chen, and G. Hu, Chemical Physics Letters 380, 78 (2003).

[63] P. Schwerdtfeger, L. N. Wirz, and J. Avery, Wiley Interdisciplinary Reviews: Computational Molecular Science 5, 96 (2015).

[64] M. Terrones, G. Terrones, and H. Terrones, Structural Chemistry 13, 373 (2002).

[65] L. Zhou and S.-Q. Shi, Applied physics letters 83, 1222 (2003).

[66] F. Cataldo, A. Graovac, and O. Ori, The mathematics and topology of fullerenes, Vol. 4 (Springer Science \& Business Media, 2011).

[67] R. Osserman, A survey of minimal surfaces (Courier Corporation, 2013). 